ÉGYPTE

monde arabe

\section{Égypte/Monde arabe}

6 | 1991

Des espaces qualifiés 2

\title{
Entre tradition et occidentalisation : l'aménagement du quartier de Hilmiyya al-Gadida au tournant $\mathrm{du} \mathrm{xx}^{\mathrm{e}}$ siècle
}

\section{Khaled 'Asfour}

\section{(2) OpenEdition \\ Journals}

Édition électronique

URL : https://journals.openedition.org/ema/439

DOI : $10.4000 /$ ema.439

ISSN : 2090-7273

Éditeur

CEDEJ - Centre d'études et de documentation économiques juridiques et sociales

Édition imprimée

Date de publication : 30 juin 1991

Pagination : 37-56

ISSN : 1110-5097

Référence électronique

Khaled 'Asfour, « Entre tradition et occidentalisation : I'aménagement du quartier de Hilmiyya al-

Gadida au tournant du xx siècle », Égypte/Monde arabe [En ligne], 6 | 1991, mis en ligne le 08 juillet

2008, consulté le 07 juillet 2022. URL : http://journals.openedition.org/ema/439 ; DOI : https://doi.org/ 10.4000/ema.439

Ce document a été généré automatiquement le 7 juillet 2022.

Tous droits réservés 


\title{
Entre tradition et occidentalisation : l'aménagement du quartier de Hilmiyya al-Gadida au tournant du $\mathrm{xx}^{\mathrm{e}}$ siècle
}

\author{
Khaled 'Asfour
}

1 Au tournant $\mathrm{du} \mathrm{xx}^{\mathrm{e}}$ siècle ${ }^{1}$, le processus de réforme qui s'était manifesté dans divers aspects de la culture égyptienne, y compris l'architecture et la planification urbaine, se poursuit au Caire. La rencontre de l'Égypte avec la culture européenne n'était pas récente. Le temps où les cultures autochtone et européenne étaient entrées en contact était déjà loin, et l'espoir que la présence étrangère ne serait qu'une expérience temporaire n'était plus de mise. Désormais la culture locale devait faire une place permanente au nouveau-venu : l'Européen. Ainsi, un processus rigoureux d'interaction se produisait, qui impliquait, entre autres, destruction, diffusion, adaptation et résistance. La critique était vive: l'Européen était perçu à la fois comme un contaminateur et comme un sauveur; l'autochtone quant à lui était qualifié en même temps de rétrograde et d'authentique. Cette période fut celle des réévaluations et des innovations.

2 Cette interaction entraîna des réponses diverses; de l'acceptation forcée à la fascination quasi religieuse, du rejet total au compromis conditionnel. Je me suis attaché à étudier l'attitude modérée, le sentiment mitigé qui prenait en compte la validité des deux composants à la fois : l'autochtone et l'étranger. Le plus souvent, les intellectuels adoptant une semblable position considéraient encore ces deux éléments comme étant diamétralement opposés. Cependant il ne s'agissait pas d'une opposition reposant sur un antagonisme foncier, sans compromis possible, mais plutôt d'une incompatibilité temporaire qui séparait «naturellement » l'ancien et le moderne. Des inquiétudes devaient certainement exister mais on s'appliquait à rapprocher les deux pôles plutôt qu'à les séparer. Comment ? Et comment cette incompatibilité a-t-elle pu 
être dépassée ? Mon étude se propose de répondre à ces questions en s'attachant à l'architecture et au développement du quartier de Hilmiyya.

Le Caire autour de 1840

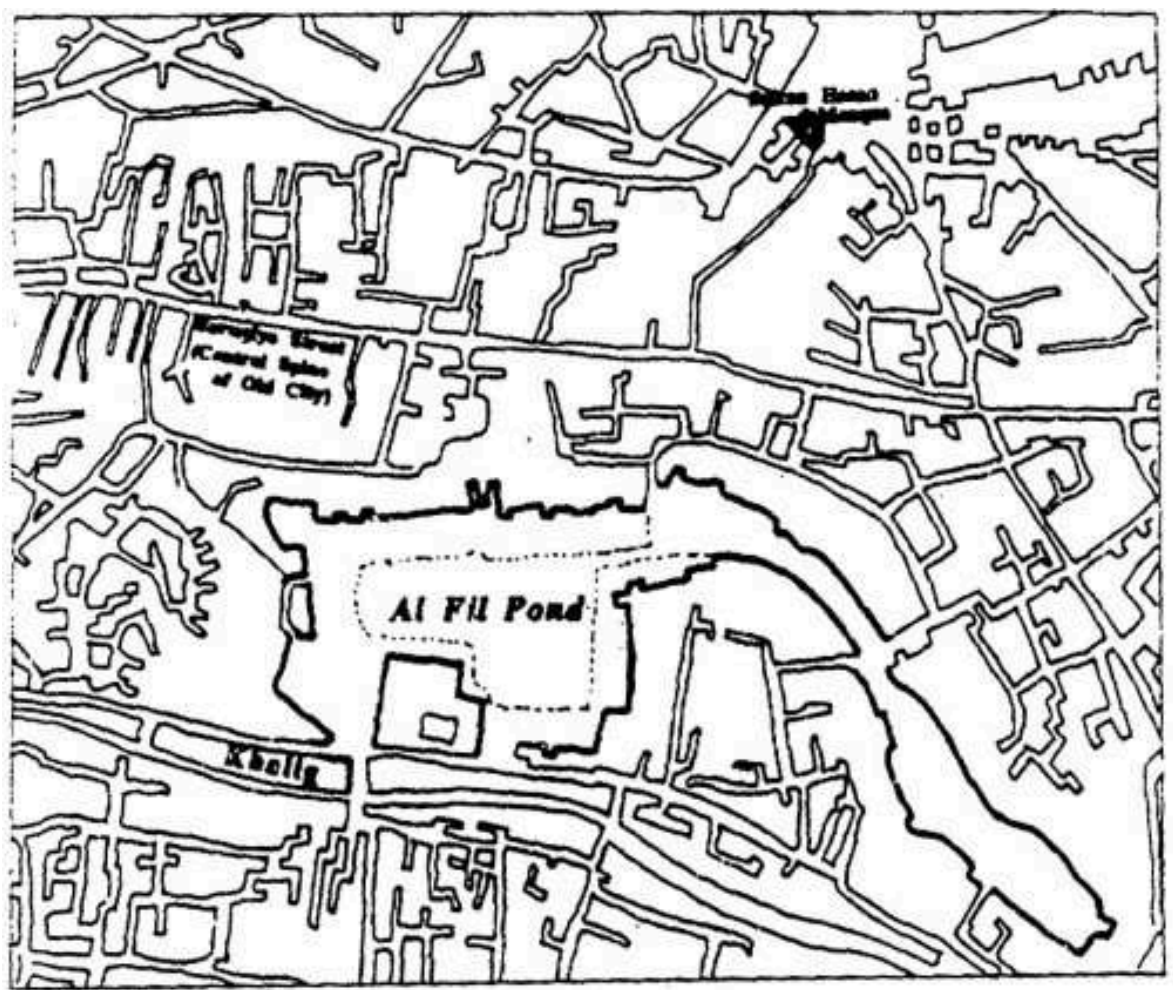

Carte de la Description montrant l'étang al-Fil en 1800

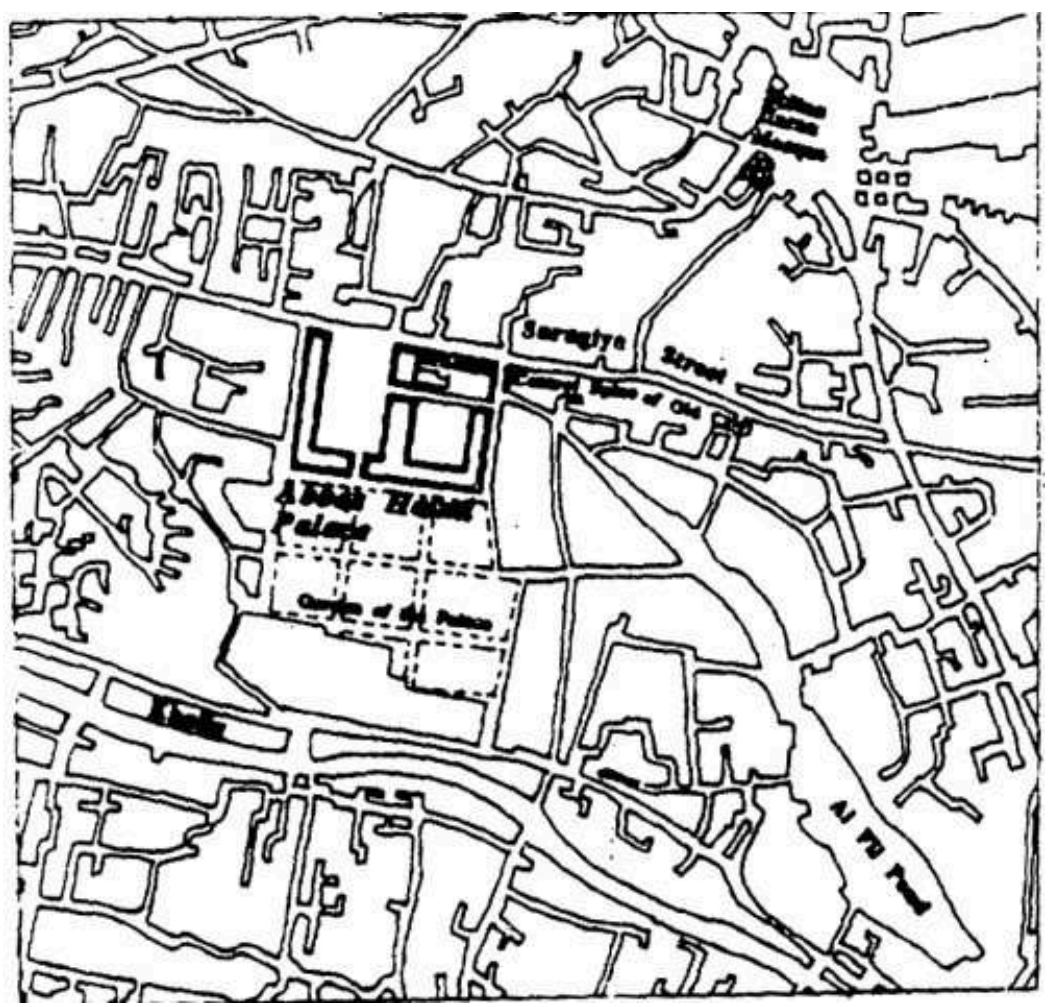


Ce quartier a été construit sur l'emplacement de l'étang al-Fil. Au xix ${ }^{\mathrm{e}}$ siècle, la famille de Mohamed Ali se rendit propriétaire de l'endroit et fit combler la majeure partie de l'étang pour y construire un palais pour l'un des princes : 'Abbas Hilmi $1^{\mathrm{er}}$. Un plan de 1840 montre le palais avec son jardin donnant sur la rue al-Mu'iz, l'axe central du Caire. La carte de Grand bey des années 1870 montre le palais et le découpage détaillé du jardin. Le palais a perdu de son importance mais a conservé son orientation par rapport à la vieille artère du Caire. De plus, le jardin a été divisé en un quadrillage d'allées qui suivent la même orientation. Par contre le boulevard Mohamed Ali, une nouvelle artère percée en $1873^{2}$, prend une toute autre direction.

4 En 1894 le jardin est réaffecté par le ministère des Travaux publics et redessiné en un tissu de rues et de parcelles à bâtir. En 1903, le palais est démembré et subit le même processus de reconversion. C'est ainsi que fleurit tout un quartier : Hilmiyya al-Gadîda.

5 Si on superpose la carte de 1896 publiée par le ministère des Travaux publics avec celle de Grand bey en 1876, on comprend quelle conception a été mise en œuvre pour transformer la structure du jardin en rues d'utilité publique. Le planificateur a choisi l'intersection entre le boulevard Mohamed Ali, artère principale du Caire moderne, et la rue al-Mu'iz, axe central du Vieux Caire. A partir de ce point, il a suivi une allée nordsud du parc qui se termine par une autre orientée est-ouest. Jusque-là il ne s'est pas contenté de respecter l'orientation du jardin, il a tout simplement suivi ses axes.

En orientant les rues selonle découpage du jardin, c'est-à-dire l'axe traditionnel d'alMu'iz, l'urbaniste devait aussi prendre en considération l'orientation du nouveau boulevard. Il résolut le problème en traçant une rue perpendiculaire à celui-ci. Pour " absorber » le décalage existant entre les deux réseaux, le moderne et le traditionnel, il eut recours à des carrefours à l'intérieur du quartier. En théorie, la rue perpendiculaire au boulevard Mohamed Ali aurait dû rejoindre les deux autres à un carrefour circulaire. Mais l'urbaniste rechercha un aménagement plus intéressant en faisant correspondre cette rue perpendiculaire à une ruelle située de l'autre côté du boulevard conduisant à l'entrée principale d'origine de la mosquée Qusun qui donne sur l'artère traditionnelle. Le résultat fut la création d'un carrefour particulier légèrement décalé par rapport au point d'intersection des deux axes. Aussi étonnant que cela puisse paraître, l'urbaniste sacrifia l'idée d'un tracé à la géométrie idéale (trois rues rayonnant à partir d'un carrefour) pour s'adapter aux conditions locales particulières. Le reste du réseau viaire est orienté soit à partir de l'axe ancien soit à partir de l'axe moderne. 
Plan de Grand Bey montrant le Palais de Abbas Hilmi $1^{\text {er }}$
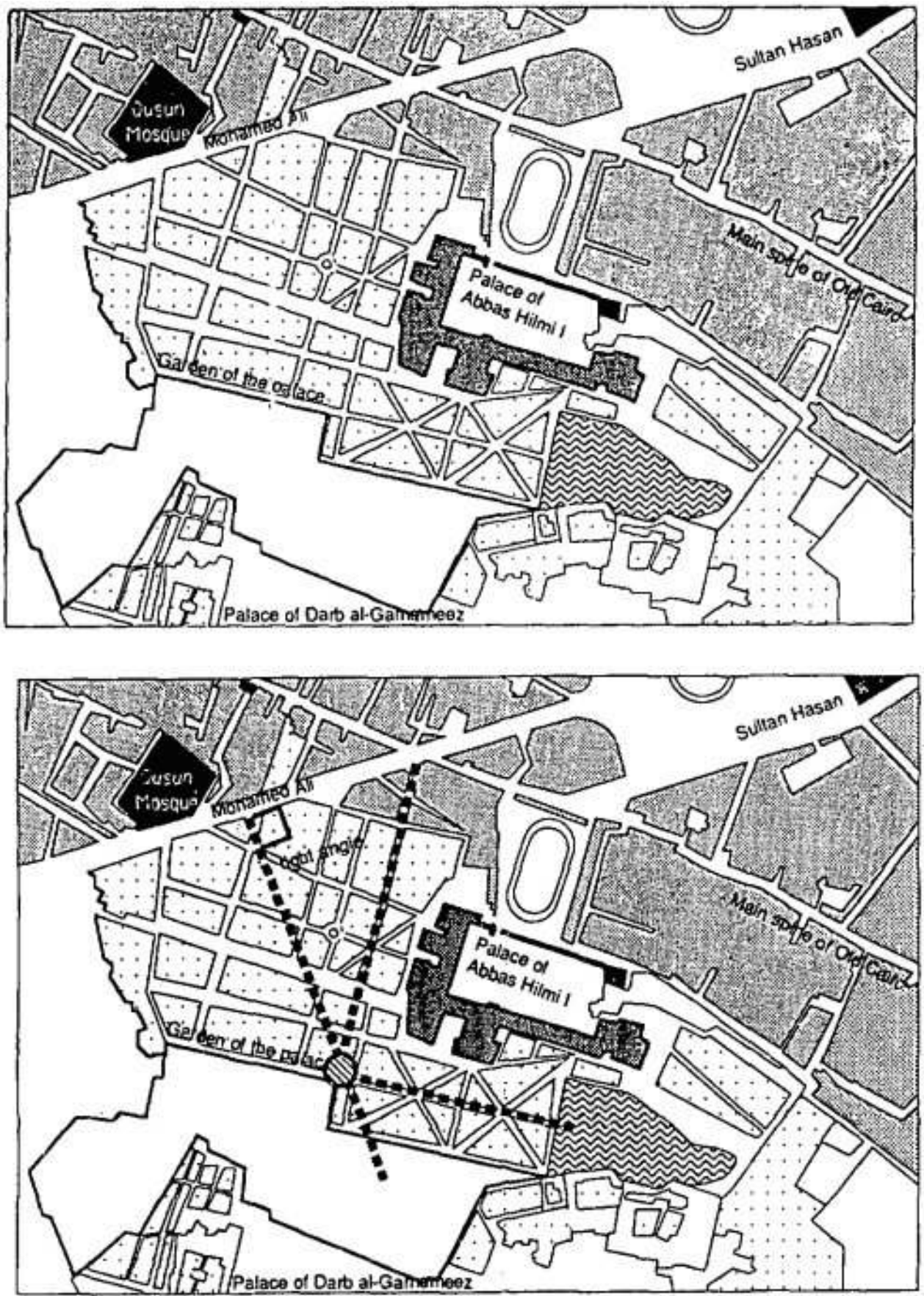


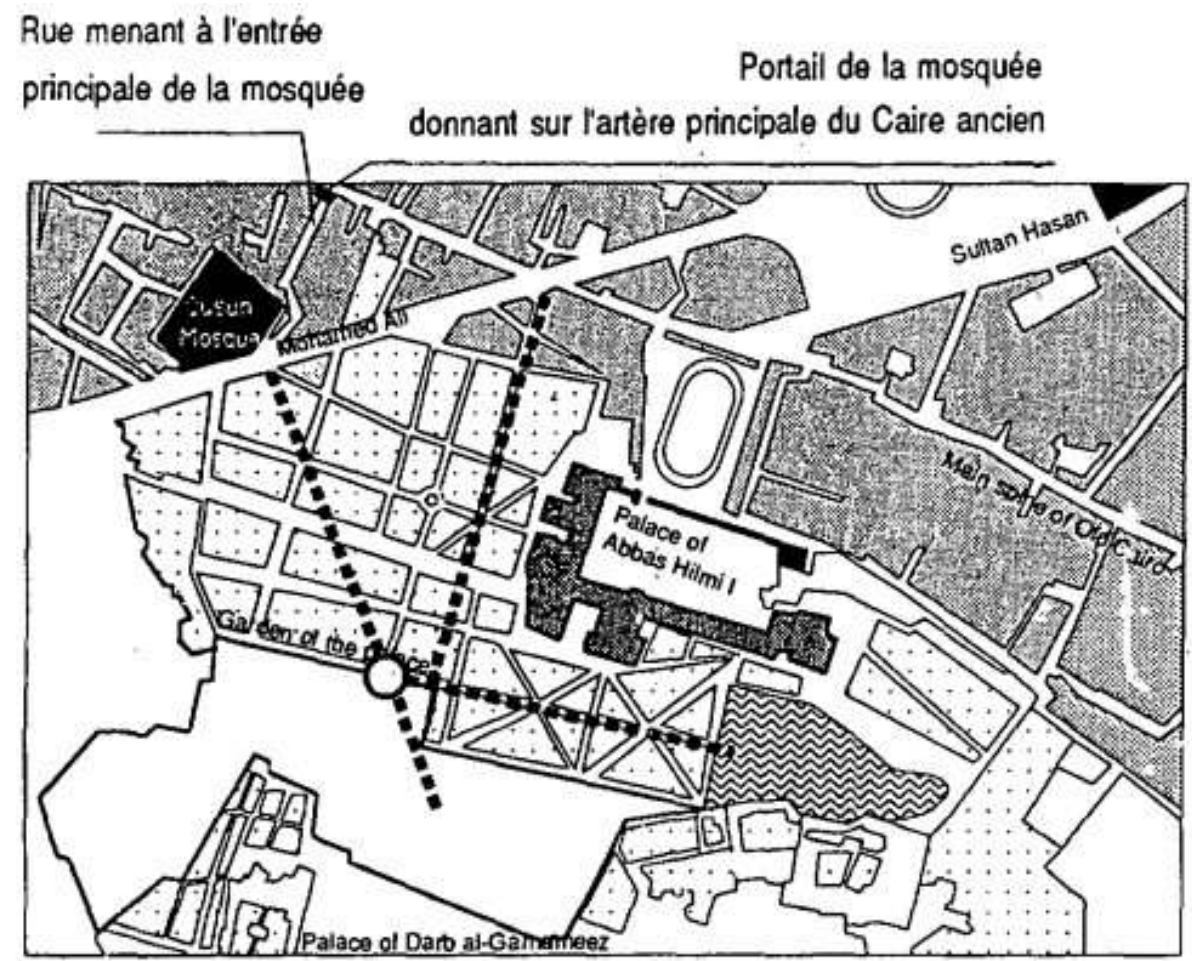

Première phase de l'aménagement

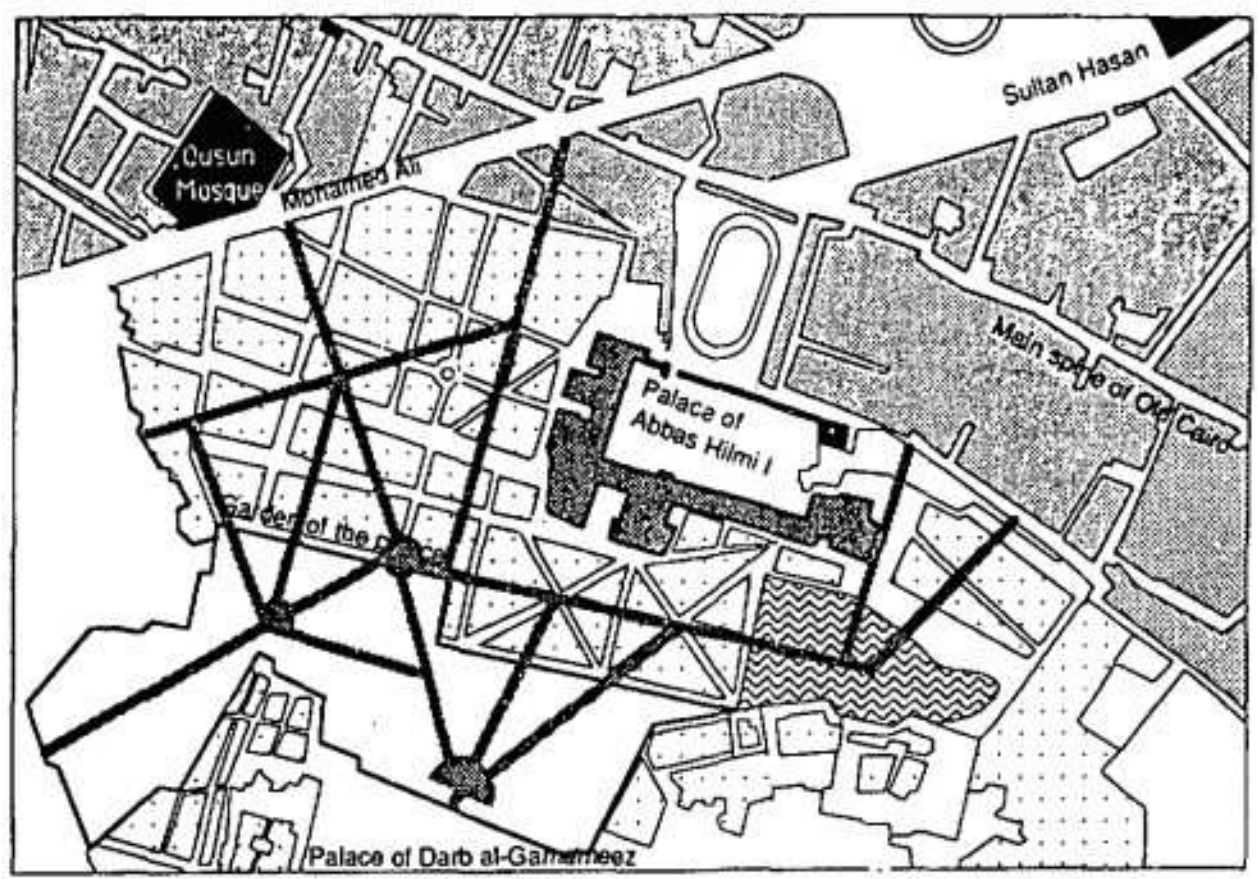


Entre tradition et occidentalisation : l'aménagement du quartier de Hilmiyya ...

6

Aménagement final
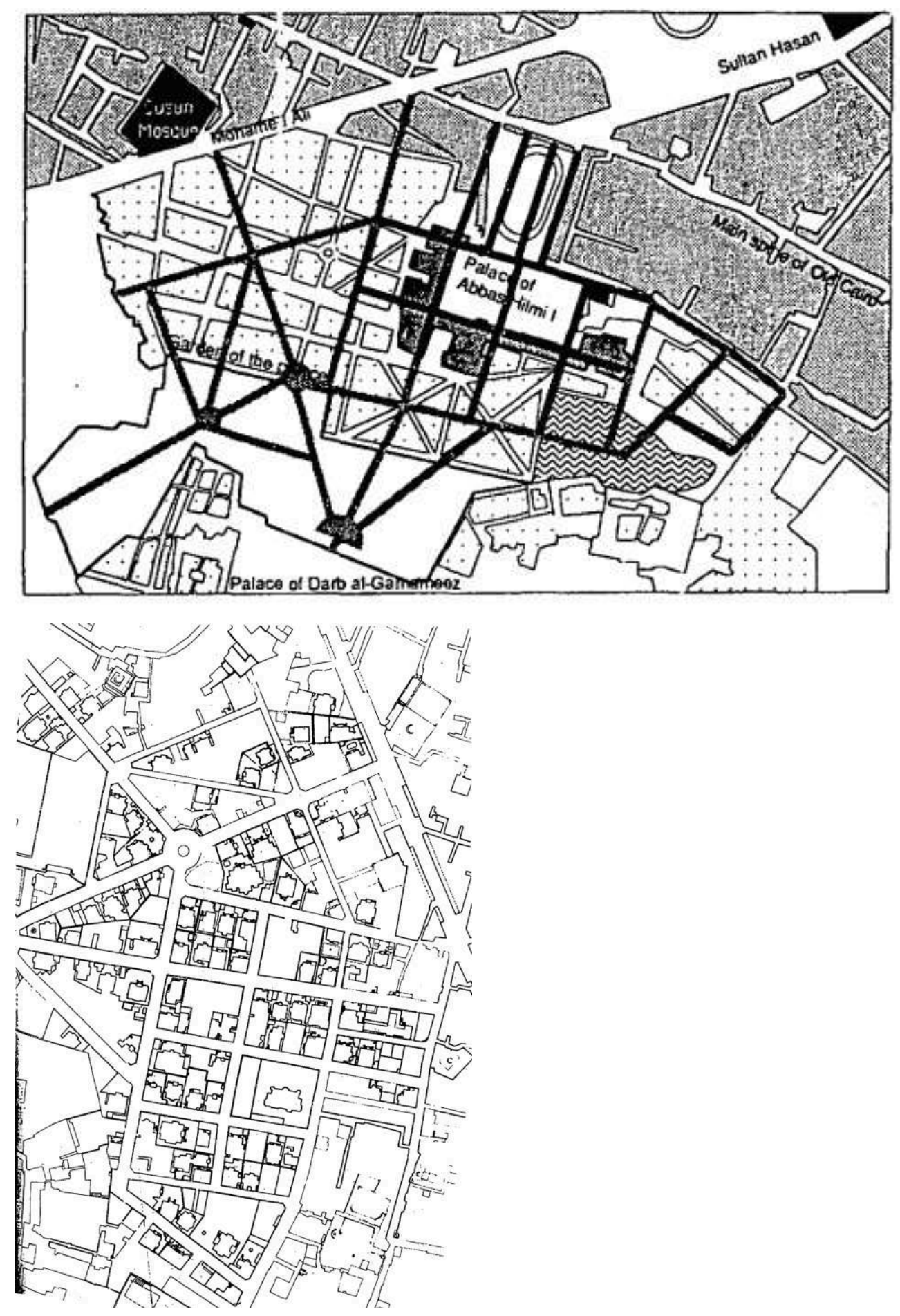

Égypte/Monde arabe, 6 | 1991 


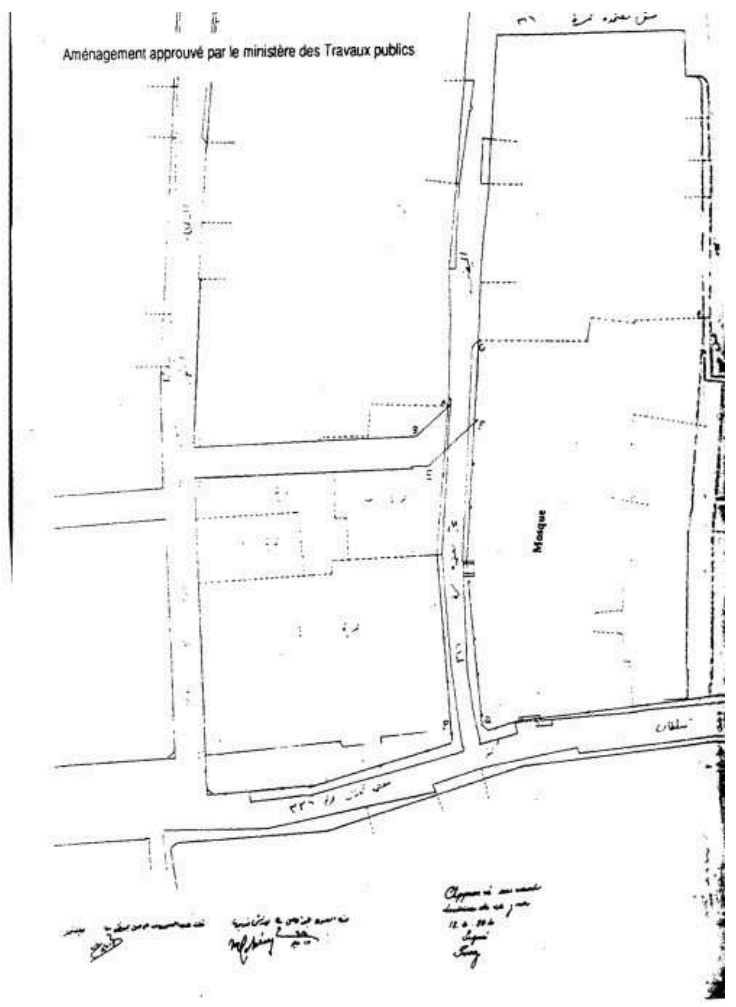

7 Dans la seconde phase, le palais fut démembré et l'urbaniste procéda de la même manière que pour le jardin en s'appuyant sur la configuration propre du palais. Il traça un nouveau réseau de rues dans la cour de celui-ci. Par la suite il procéda à de nouvelles divisons. La configuration finale de Hilmiyya est donc un réseau de rues dont certaines correspondent à l'artère moderne représentée par le boulevard Mohamed Ali et d'autres à la vieille artère qu'est al-Mu'iz. L'idée de concilier l'ancien et le moderne, l'européen et l'autochtone, est donc l'une des bases du plan de Hilmiyya. Pour célébrer cette nouvelle conception, le ministère des Travaux publics, qui était situé dans les environs, décida de donner au carrefour reliant le réseau traditionnel et le réseau à l'occidentale le nom du quartier.

8 L'œuvre du ministère reflète une attitude favorable à l'européanisation. La composante européenne n'était plus considérée comme un élément totalement étranger à la culture locale et devait donc s'imposer comme dans le cas du boulevard Mohamed Ali. L'alignement parfait de la rue, perçant à travers la partie la plus dense du tissu urbain sur une longueur d'un kilomètre, dénote une approche qui entre en conflit avec le tissu traditionnel. Le tracé géométrique du boulevard ne tient pas compte de celui-ci. On jugeait que la Vieille Ville, avec ses rues irrégulières, ses impasses et ses allées sombres, n'était ni hygiénique, ni appropriée aux nouveaux systèmes de transport et qu'elle ne correspondait pas à l'image grandiose que l'on voulait donner du Caire.

Cependantau tournant $\mathrm{du} \mathrm{xx}^{\mathrm{e}}$ siècle l'approche n'est plus la même. Les politiques d'aménagement urbain reflètent une attitude moins extrême vis-à-vis des deux courants, le traditionnel et l'européen, et une méthode d'intervention plus subtile est adoptée dans des situations ponctuelles. (...)

Cette attitude plus modérée prévaut en effet non seulement dans la construction de la nouvelle ville mais également dans le réaménagement de la vieille Ville. En 1904, le 
ministère des Travaux publics reçut une demande émanant du ministère des Waqfs concernant la restauration de la mosquée du Sultan al-Hanafî et l'embellissement des rues avoisinantes. Lorsqu'on examine le plan approuvé par le ministère, on se rend compte que la politique de réaménagement du quartier impliquait l'extension d'une rue latérale pour qu'elle débouche sur une artère principale. D'autre part cela impliquait également la création d'une liaison entre la rue qui buttait sur un côté de la mosquée et une autre. L'objectif principal était de rendre ainsi la mosquée plus visible et accessible. Le ministère fit également part de son désir que les rues voisines soient régularisées (ta'dîl muntazim) afin qu'elles correspondent mieux à la valeur de la mosquée ${ }^{3}$. Cette politique signifie, dans ce cas précis, non pas la création d'alignements indéfiniment parallèles mais plutôt une étude détaillée des rues, section par section, rive après rive, de manière à voir quelles portions de façade devaient être préservées et éventuellement créées pour obtenir un aspect suffisamment régulier tout en causant un minimum de dommages. Ainsi on aboutit à des rues plus régulières avec des ruelles élargies se terminant en $\mathrm{T}$, en harmonie avec le contexte, au lieu d'en effacer le caractère traditionnel pour répondre à une géométrie idéale.

11 L'application de systèmes occidentaux au milieu local se généralisa dès la fin du XIX siècle. Elle ne se limitait pas à l'aménagement urbain mais concernait également l'architecture.

12 Le développement d'un enseignement moderne en Égypte favorisa la mise en place de cette politique. Mohamed Ali, déterminé à faire de l'Égypte un État puissant, s'attacha à créer des écoles où étaient enseignées les sciences occidentales. Tous ces établissements avaient pour but l'édification de l'armée et devaient donc apporter la preuve du profit réel qu'ils pouvaient lui offrir ${ }^{4}$. On favorisa par conséquent les disciplines pratiques en raison de leur application immédiate. L'unique critère pour l'enseignement était le rapport efficace qu'il était possible d'établir à cette époque entre théorie occidentale et pratiques locales, technologie moderne et production intérieure. Cette philosophie continua de prévaloir même après la dissolution presque complète de l'armée en $1882^{5}$.

13 Dans cette perspective, il convient de replacer l'architecture dans le contexte de la Muhadiskhâna (école polytechnique) : c'était l'institution officielle pour l'enseignement de l'architecture en Égypte au XIX et au début du Xx ${ }^{e}$ siècle $^{6}$.

14 La Muhandiskhâna a été fondée dès la première moitié du XIXème siècle pour former les étudiants dans le domaine de l'artillerie et du génie maritime, des ponts et chaussées, des mines, etc. Il était clair à l'époque que toutes ces spécialisations nécessitaient une connaissance des mathématiques et des sciences physiques ${ }^{7}$. Même après les réformes

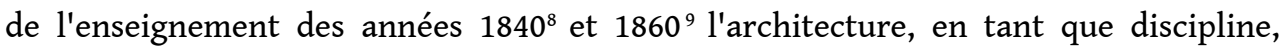
continua de n'être qu'une partie d'un programme scolaire très largement orienté vers la technologie. Celui-ci comportait la géométrie, l'algèbre, les statistiques, la mécanique, la physique, la chimie, etc. ${ }^{10}$ Dans ce contexte, l'architecture était simplement considérée comme une discipline technique parmi d'autres visant à enseigner les méthodes de construction et non pas l'art du projet. En conséquence, la discipline était coupée de son cadre théorique.

15 La théorie de l'architecture était enseignée à l'École des Beaux-Arts de Paris qui, dans l'Europe du XIX ${ }^{e}$ siècle, exerçait la plus forte influence et était la plus prestigieuse. La pensée maîtresse de l'école s'organisait autour de la notion platonicienne d'Idée. Celeci conçoit la beauté parfaite comme une entreprise métaphysique qui ressortit 
exclusivement au monde intelligible et qui accède à la réalité en prenant la forme d'un objet selon des lois universelles qui trouvent leur fondement dans la nature même. Ces lois sont la symétrie, l'axialité, les proportions, la hiérarchie, l'ordre et l'harmonie, c'est-à-dire tout ce qui en architecture correspond à l'expression "la belle nature ", notion qui avait été développée dans l'Antiquité par Vitruve. Un objet est beau en fonction de la quantité de beauté (cette notion sublime et spirituelle) que la matière incorpore $^{11}$. Cette notion ne peut pas être complètement atteinte, mais ce que les artistes peuvent faire, c'est tendre vers la perfection par leur maîtrise, intellectuelle et artistique, de la matière. Pour cela, l'âme doit être habituée à la vue de belles oeuvres. Aussi aux Beaux-Arts l'étudiant était-il formé à rechercher l'inspiration dans ce qui était reconnu comme les chefs-d'œuvres de maittres tels que Vitruve et Palladio ${ }^{12}$. Il n'était pas seulement encouragé à étudier les édifices historiques majeurs de Rome et d'Athènes, mais également les projets théoriques des meilleurs élèves plus âgés («les Anciens ») de l'école ${ }^{13}$.

Pour chaque projet, il commençait par concevoir le «parti » ou schéma d'organisation du bâtiment. Il devait aussi définir le style convenant le mieux au programme de construction. L'étudiant brillant était celui qui savait utiliser au mieux ce qu'il avait appris des Maîtres, l'étude des dispositions et des proportions. Il devait également être capable d'harmoniser au plus haut degré le "parti" et le programme de construction, l'école enseignait aux étudiants une attitude puriste à l'égard de l'architecture, indifférente à son environnement.

17 A l'inverse, dans les écoles polytechniques l'architecture était une science pratique qui répondait aux conditions locales. C'est le programme de ce type d'école qui fut adopté au Caire. L'architecture qui était enseignée en cinq ans aux Beaux-Arts fut réduite à un ou deux cours : aussi dut-elle être considérablement simplifiée. Tout l'esprit créatif que l'on développait aux Beaux-Arts chez les étudiants pour essayer d'atteindre la notion sublime de beauté, était étouffé dans le programme de l'école polytechnique par l'utilisation d'ouvrages encyclopédiques codifiant et indexant des types de programmes de construction et des styles d'architecture. La méthode permettait aux étudiants d'appréhender les formes classiques comme des éléments modulaires standardisés. Dans le but d'élaborer des programmes de construction, ils apprenaient à assimiler ces éléments sur un papier quadrillé au moyen de principes de permutation et de règles de symétrie comme pour assembler les pièces d'une machine ${ }^{14}$. Ainsi dépourvu de théorie, l'enseignement de l'architecture à l'école polytechnique consistait à assurer une formation pratique qui répondait par définition aux besoins du pays. Cela fut le cas de l'école établie au Caire. Les professeurs, qu'ils fussent européens ou égyptiens, étaient principalement des diplômés de l'École polytechnique.

18 Il n'est donc pas surprenant que le règlement de l'école du Caire prescrivît une formation pragmatique. Dans les lois de 1902 et de 1908, les articles 13, 14 et 15 stipulent que chaque cours doit comprendre un certain nombre d'heures destinées à l'application. Ainsi, les étudiants en architecture étaient-ils tenus de suivre la réalisation de bâtiments et de noter leurs observations. D'autre part on les envoyait effectuer des relevés dans différents sites. En outre, les manuels destinés aux étudiants, tels ceux d'Ali Moubarak ${ }^{15}$, décrivent de façon extensive les matériaux et la construction. Ils ne présentent qu'un seul des cinq ordres architecturaux, l'ordre toscan, en raison de sa relative simplicité. Tout ce que les étudiants devaient en connaître étaient les équationsutilisées pour obtenir les proportions correctes. La 
relation de cet ordre avec les divers programmes de construction, les théories liées à celui-ci et son origine n'étaient jamais abordées.

Exemple de traitement de façade : la villa Delbrour Choukri à Hilmiyya

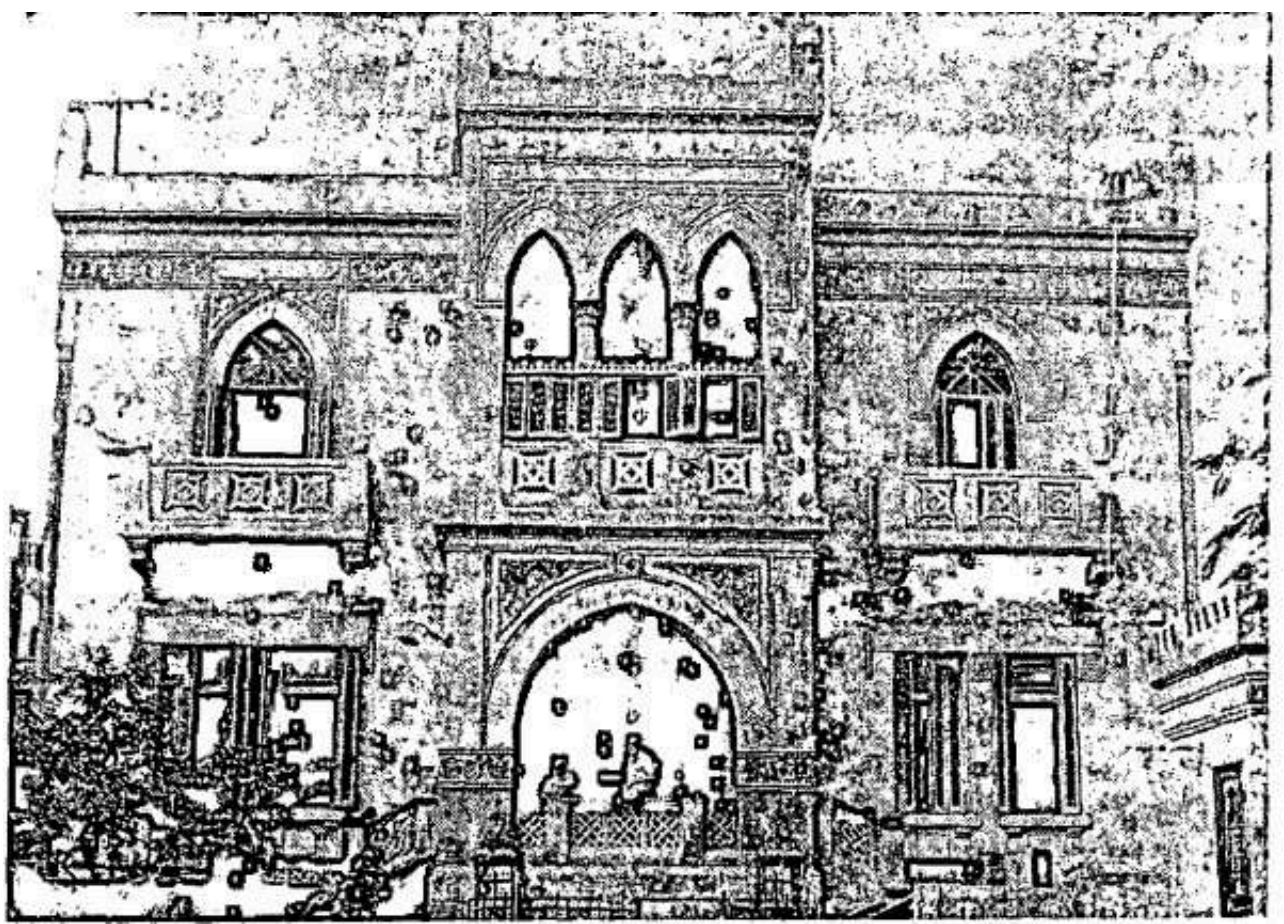

Décor de façade à Hilmiyya

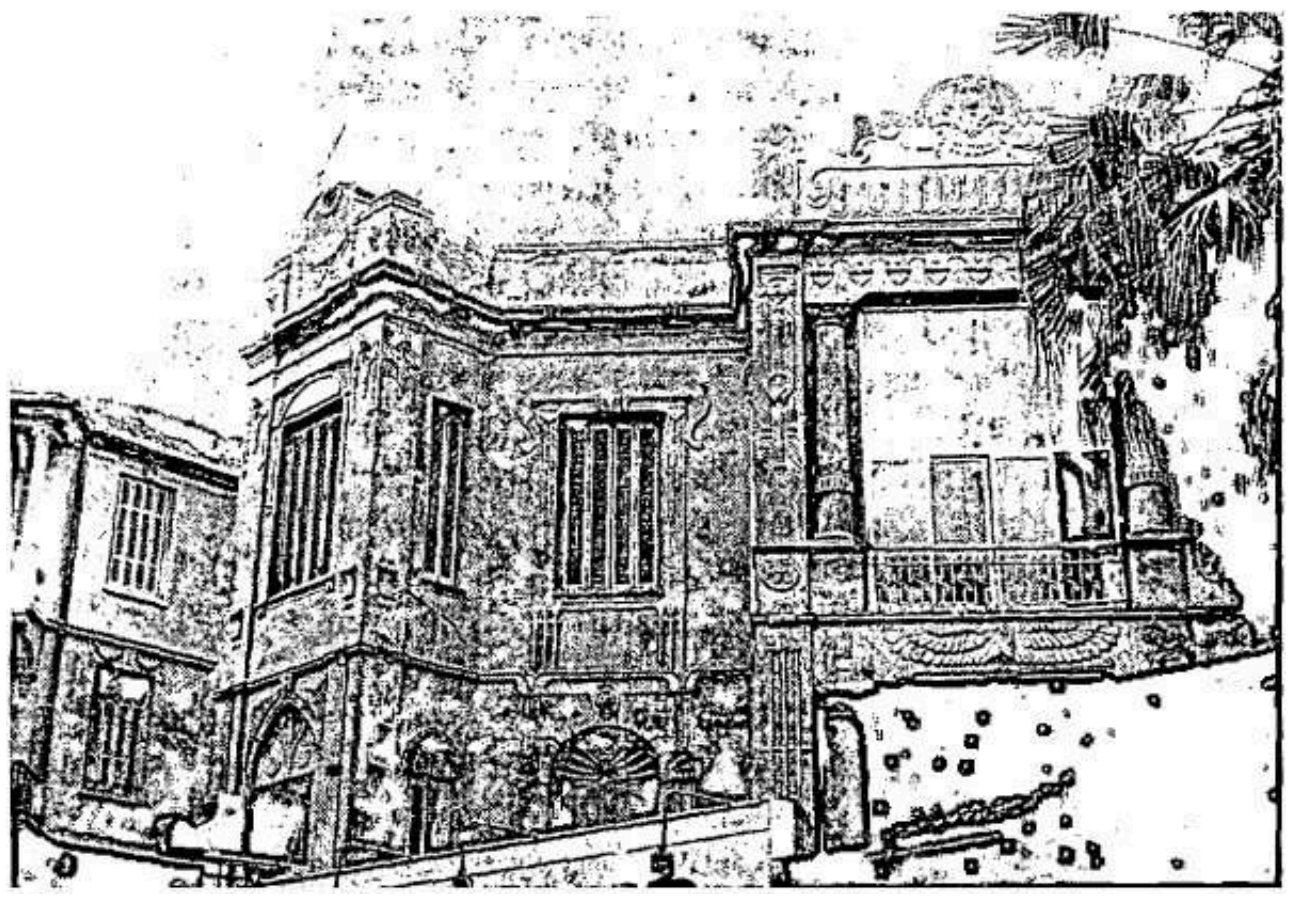

Vingt ans plus tard, en 1897, un autre professeur, Muhammad 'Aref, écrivit un manuel à l'intention des étudiants en architecture de cette même école ${ }^{16}$. Comme Ali Moubarak, il voit dans l'architecture une discipline purement technique qui enseigne les méthodes 
de construction des bâtiments. Un des critères de sa définition de l'architecture est la réponse aux conditions locales ${ }^{17}$. Pour lui, les bâtiments sont composés d'éléments qui sont assemblés et qui sont gouvernés par des lois mécaniques de régularité et de symétrie. Cette vision explique l'attitude dominante vis-à-vis de l'architecture traditionnelle de l'époque. Celle-ci est considérée comme un ensemble d'éléments qui peuvent être théoriquement réassemblés de manière régulière et symétrique lors de la conception de nouveaux édifices. Il conçoit la beauté comme l'expression, à travers leur aspect intérieur et extérieur, de la fonction des bâtiments. Dans ce contexte le décor n'a aucun rapport avec le programme de construction proprement dit, il est juste un traitement de surface qui est du seul ressort de l'artisan et qui a pour but de refléter la richesse du propriétaire. Dans cette école, l'étudiant en architecture se contentait d'acquérir un recueil de modèles proposant des planches d'ornements. Ainsi le livre de Bourgoin (1873) sur les motifs islamiques présentait-il un intérêt particulier pour 'Aref qui en fit un résumé dans son manuel. Aussi pouvons-nous comprendre le décor de certaines maisons de Hilmiyya comme des séries de "planches » disparates sans lien avec le langage formel de l'édifice.

En 1868 la Muhandiskhâna est transférée dans le palais du Prince Mustafa Fâdil à Darb al-Gamamiz, un quartier situé au sud de Hilmiyya. Ainsi comprend-on mieux que l'approche architecturale de cette école y ait trouvé sa pleine expression.

A cette époque, la fonction du quartier était principalement résidentielle. Clerget, dans sa classification sociale du Caire, décrit la société de Hilmiyya comme étant à moitié indigène et à moitié européenne ${ }^{18}$. Cela n'est pas surprenant, puisqu'elle était composée de membres de l'élite cultivée de la ville dont certains avaient même été envoyés en mission d'étude en Europe. Nous y trouvons un grand nombre de hauts fonctionnaires, de réformateurs et de critiques littéraires connus. Mais ce qui caractérise Hilmiyya en tant que foyer culturel est le pourcentage exceptionnellement élevé d'écoles situées à Darb al-Gamamiz. Il y avait une école préparatoire, une école secondaire, la Muhandiskhâna, un institut d'égyptologie ainsi que l'École d'administration et de langues (al-Afsun) qui plus tard devint l'École de droit ${ }^{19}$. 


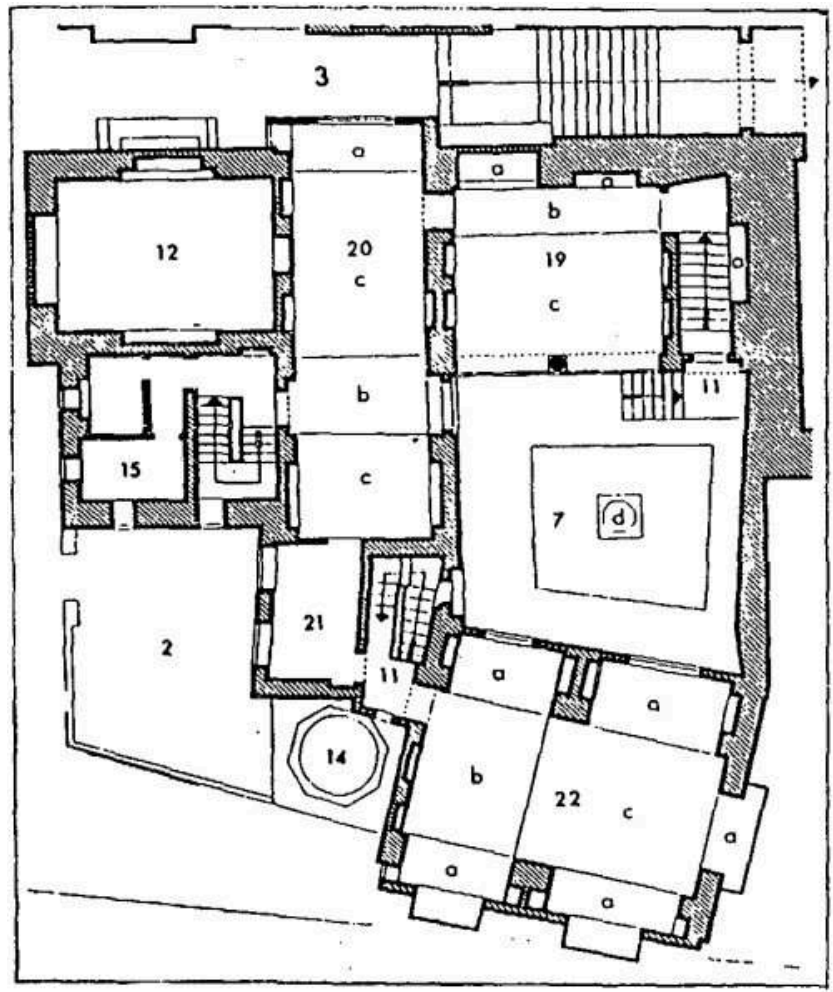

L'atmosphère "pédagogique » de Hilmiyya donna l'idée au ministère des Travaux publics de baptiser les nouvelles rues tracées dans le quartier du nom de personnalités du domaine de l'enseignement: Ali Moubarak (ministre du Conseil des écoles), Riyad Pacha (ministre de l'Éducation du cabinet de 1873), 'Ali ibrahîm (ministre de l'Éducation du gouvernement de 1879-1881) et 'Abd Allah Fikri (ministre de l'Éducation du cabinet de 1882). Il existe également une rue des Écoles (al-Madâris). Plus important encore est le fait qu'on donna à la place semi-circulaire qui relie Darb al-Gamamiz à Hilmiyya le nom du prince Mustafa Fâdil (frère cadet du khédive Ismaiil), qui avait fait expressément don de son palais pour créer la Muhandiskhâna à Darb al-Gamamiz.

Les habitants de Hilmiyya vivaient dans des maisons qui combinaient aspirations européennes et dispositions traditionnelles. Le plan de 1911 est très révélateur : la plupart des maisons comprennent une construction distincte à laquelle on accède par quelques marches, qui est située le plus près possible de la porte d'entrée du jardin ou même possède une entrée différente. C'est le salâmlik, une salle de réception destinée à accueillir les inconnus ou les connaissances desexe masculin. Dans les demeures cairotes $\mathrm{du} \mathrm{xvIII}^{\mathrm{e}}$ siècle, le salâmlik était intégré à la demeure, il pouvait prendre la forme d'une qấ'a (salle de réception) reliée à un maq'ad (loggia), située au premier étage et/ou d'une qấa située au rez-de-chaussée (mandara). Il convient de noter qu'en général dans la maison traditionnelle les salles de réception étaient des espaces importants et que leur conception faisait l'objet d'un soin tout particulier: non seulement elles devaient bénéficier de la meilleure orientation mais, contrairement aux pièces de service, leur forme était régulière.

Dans la maison Kiridliyya par exemple, l'axe de la qấ' $a\left(n^{\circ} 22\right)$ a été déplacé de manière à s'aligner sur celui de la rue et à respecter l'orientation cardinale. La cage d'escalier $\left(n^{\circ} 11\right)$ et la courette $\left(n^{\circ} 7\right)$ servent à «absorber » ce déplacement. Il y a donc une 
hiérarchisation dans la conception du plan; on privilégie les pièces de réception tandis que la forme des pièces de services est tributaire des particularités du terrain. Plus important encore de notre point de vue est le fait que la maisonest perçue comme un ensemble d'espaces qui sont des entités autonomes et sont reliés entre eux par l'addition de partis architecturaux indépendants de l'organisation finale de la maison.

Dans les demeures de Hilmiyya, les principes auxquels obéit la disposition interne des espaces sont complètement différents : elles sont conçues sur des modèles de villas italiennes. Le plan est tripartite. La partie médiane comprend un hall central (appelé également sâla en dialecte égyptien) flanqué de deux séries de pièces. Ce hall est mis en valeur par l'adjonction d'une terrasse, d'un porche ou d'une baie vitrée. Les espaces dans ce cas perdent leur autonomie au profit d'un agencement final gouverné par la régularité et la symétrie. De plus, dans cette conception qui ne procède plus par addition, le traitement géométrique de toutes les parties de la maison est identique.

Villa Abdul Latîf - exemple de dédoublement du salâmlik

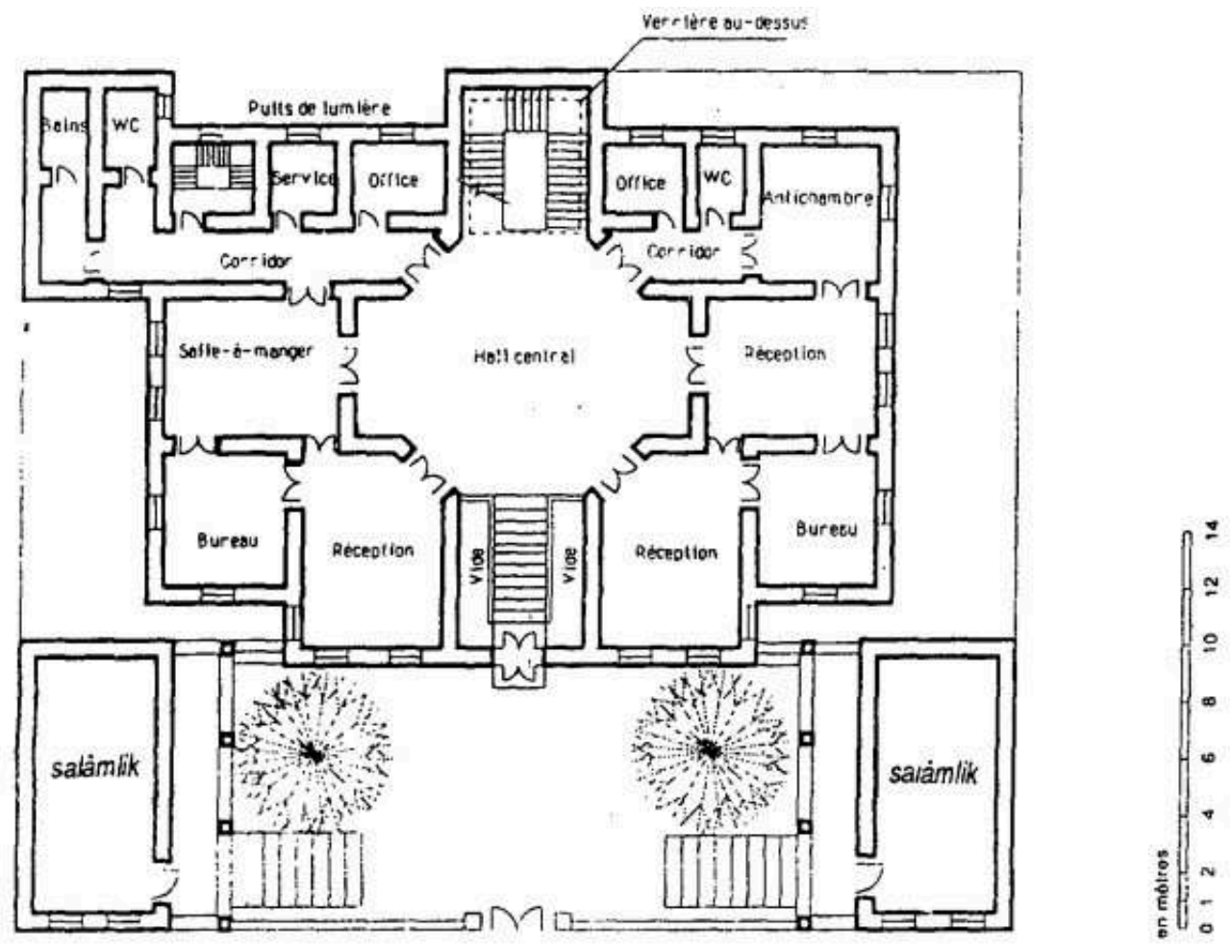




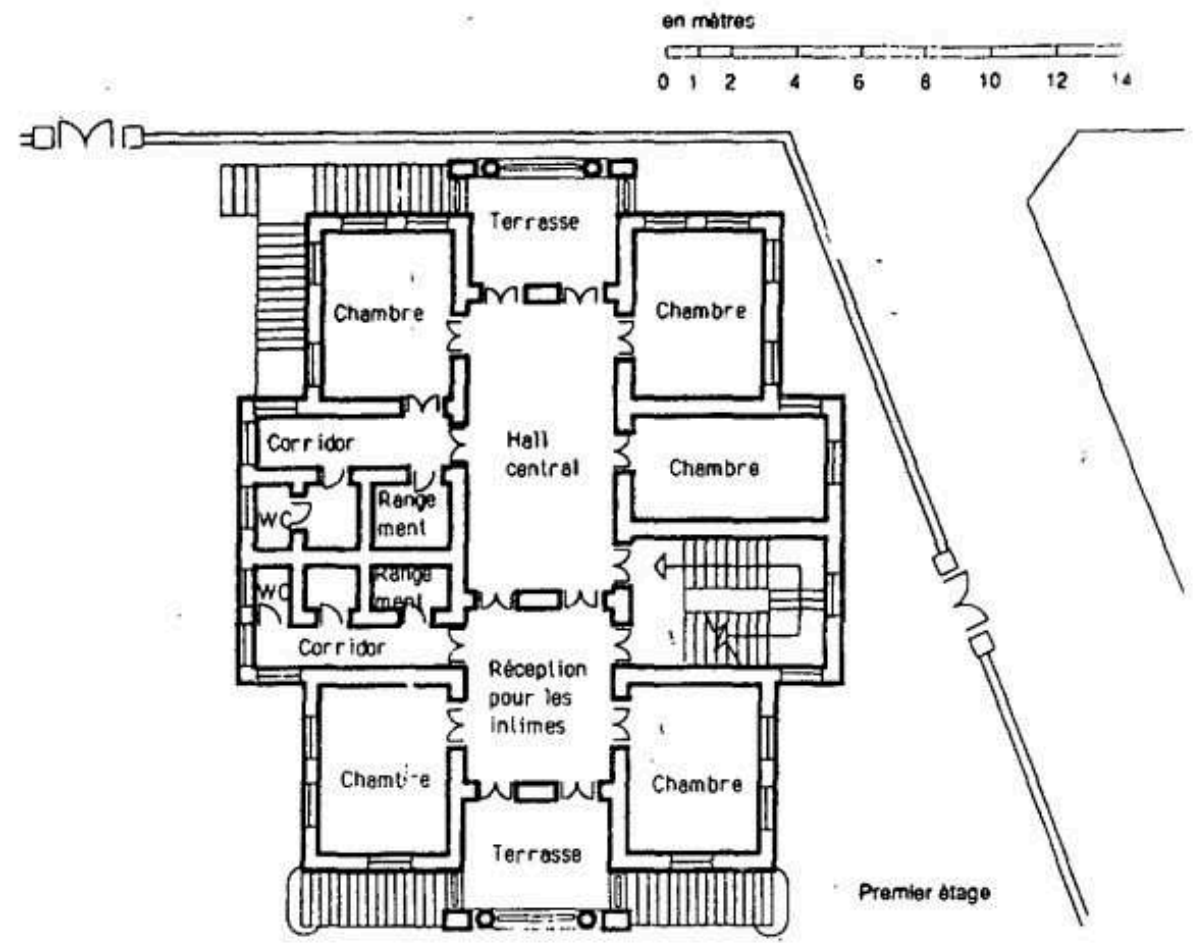

Villa de Nasim Pacha

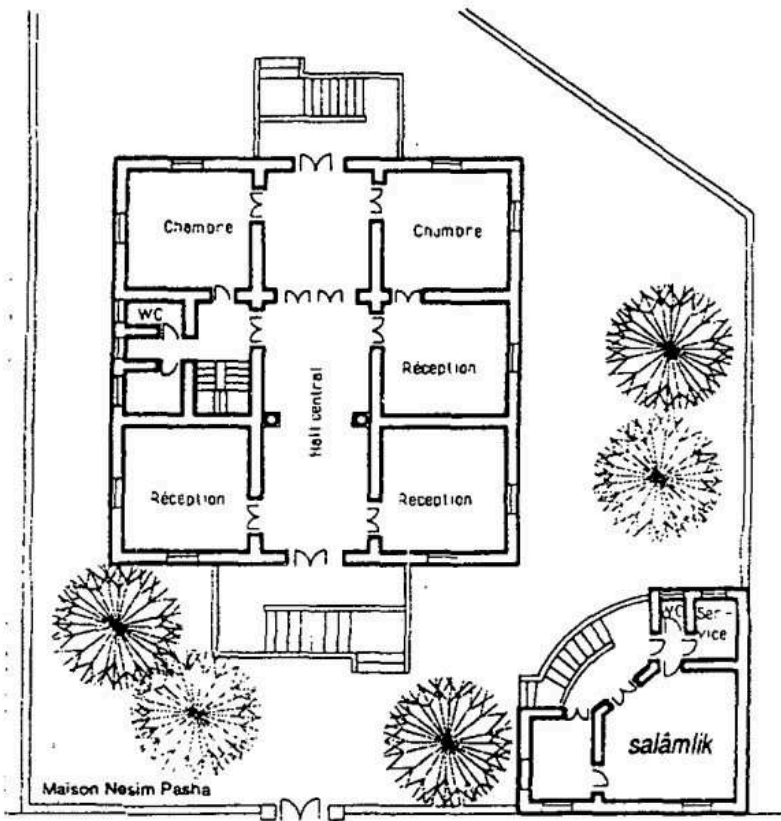

Rez-do-chanesson

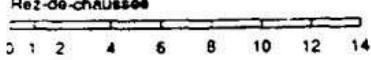

Malgré le changement radical qui s'est opéré, d'une demeure traditionnelle à une demeure «italianisée », un subtil processus d'appropriation se développe. Dans la 
typologie traditionnelle de l'habitat, c'est la séparation verticale entre vie publique et vie privée familiale qui domine et cette disposition a été retenue à Hilmiyya. Cependant, il était difficile de maintenir l'irrégularité traditionnelle du plan, en particulier l'entrée en chicane qui servait dans le passé à préserver l'intimité de la maison.

Au tournant du siècle, les rigueurs de la symétrie axiale et le dessin en trois parties n'autorisaientpas le maintien d'une telle entrée. A cet égard, les deux conceptions, l'ancienne et la nouvelle, n'étaient pas conciliables. Cependant, si le modèle étranger avait été appliqué complètement, l'intimité de la maison aurait été violée. La société aurait rejeté la disposition consistant en un hall central directement situé derrière le porche d'entrée et flanqué de pièces, sans espace intermédiaire. Par conséquent, malgré l'adoption d'un plan étranger, on continua d'appliquer le système du salâmllik. En combinant ainsi les systèmes traditionnel et nouveau, il était possible de recevoir un visiteur dans une pièce qui conservait son importance traditionnelle. Elle pouvait soit faire partie de la maison soit être située à l'extérieur. Mais dans tous les cas, l'entrée du salâmlik devait être distincte de celle du hall central. Ainsi le visiteur ne dérangerait-il pas l'intimité de la famille en pénétrant dans celui-ci. Le nouveau traitement architectural, du salâmlik montre que l'exclusion du visiteur étranger de la vie familiale - une pratique sociale traditionnelle - persistait dans la nouvelle forme d'habitat.

Cette pratique dura pendant un siècle au Caire, le refus obstiné d'altérer le plan italien étant aussi fort que la demande insistante d'un salâmlik. Une exigence, représentant la tradition, ne pouvant aller sans l'autre, symbole de modernité. La coexistence des deux est révélatrice de la façon dont l'acceptation de l'Occident constitue une base pour le changement, tout en conservant des systèmes de valeurs conventionnels.

Cette attitude rejoint la théorie de la Réforme qui avait été conceptualisée par des critiques de la société tels qu'al-Muwelhi et Muhammad 'Abdu avec les notions de «forme» et de «contenu». Dans le contexte égyptien, c'est l'émergence d'un "contenu» local associé à une "forme» étrangère qui valorisait le processus du développement, à condition que cette forme satisfasse les aspirations nouvelles à une amélioration des standards de l'efficacité et, partant, de l'image de soi.

\section{NOTES}

1. Cette recherche a été menée grâce à une bourse de l'American Research Center in Egypt (ARCE), qui l'a publiée en anglais dans son Journal ( $n^{\circ}$ 150-151 -1990). Traduction en français : CEDEJ.

2. Moubarak A. Pacha, 1887, Al-khitat al-tawfiqiyya al-gadida, Boulaq, vol. III, p. 69.

3. Documents d'archives, Conseil des ministres - Ministère des travaux publics - Equipement, Le Caire 8/8/C (lettre gim), dossier : «Rénovation de la mosquée du sultan Hanafi et confiscation de 5 maisons dans le voisinage » (20 juin 1904).

4. Heyworth-Dunne J., 1939, An introduction to the history of education in modern Egypt, Londres, rééd. 1968 ; p. 223. 
5. Les statuts de l'Université du Caire précisaient, à l'article 1, que LE but de celle-ci était d'établir un enseignement semblable à celui dispensé en Europe, dans la mesure où il était applicable en Égypte (al-Omrân 9, juin 1908, n³ 366, p. 573).

6. L'École des Beaux-Arts fut fondée en Égypte en 1908, mais ne devint officielle qu'en décembre 1913 (Al-Gabakhengi M., in : 1973, Bibliographical guide for Arab cultural values, Le Caire, GEBO, p. 292-293).

7. Heyworth-Dunne J., op. cit., p.196.

8. Heyworth-Dunne J., ibid. p. 239. 240.

9. Heyworth-Dunne J., ibid. p. 352-3.

10. Heyworth-Dunne J., ibid. p. 199.

11. Panofsky E., 1960, Idea, a concept of art theory, p. 30.

12. Egbert R., 1980, "Theory of design ", The Beaux-Arts tradition in French architecture.

13. Levine N., 1982, "The compétition for Grand Prix in 1824, case of study for architectural education at École des Beaux-Arts ", The Beaux-Arts and 19th century French architecture, p. 83-94.

14. Frampton K., 1980, Modem architecture, a critical history, p. 15.

15. Moubarak A. Pacha, 1882, Tazkirat al'muhandisîn wa tabsirat al-râghibîn (Mémento et instructions pour les étudiants).

16. 'Arif M., 1896, Khulâsat al-afkâr fí fan al-mi'mâr,, Introduction de la troisième partie, Le Caire.

17. Sami A., 1917, Al-t'alîm fî Misr.Le Caire, p. 98.

18. Clerget M., 1934, Le Caire, Le Caire, vol. 1, p. 266.

19. Heyworth-Dunne J., op.cit., p. 354-355.

INDEX

Mots-clés : cartographie, géographie, histoire, Le Caire, urbanisme

\section{AUTEUR}

\section{KHALED ‘ASFOUR}

MIT Department of Architecture 